\title{
Do technically efficient surgeons continue working at a university hospital?
}

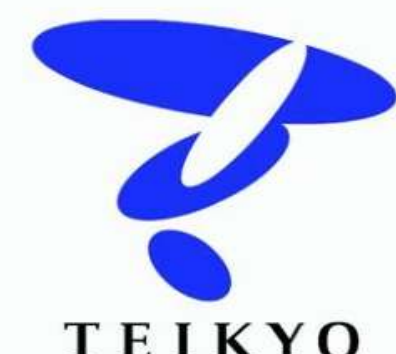

\author{
Yoshinori Nakata MD MBA ${ }^{1,2,3}$, Yuichi Watanabe MS $\mathrm{MPH}^{4}$, Sayaka Horiuchi MD MPH², \\ Hiroto Narimatsu MD PhD 5 , Hiroshi Otake MD MBA ${ }^{6}$, Tomohiro Sawa MD PhD ${ }^{3}$ \\ ${ }^{1}$ Teikyo University Hospital; ${ }^{2}$ Teikyo University Graduate School of Public Health; ${ }^{3}$ Teikyo University Medical \\ Information \& System Research Center; ${ }^{4}$ Waseda University Graduate School of Economics; ${ }^{5}$ Kanagawa Cancer \\ Center Research Institute; ${ }^{6}$ Showa University School of Medicine, Japan.
}

Objective: Recruiting and retaining technically efficient surgeons are the keys for the hospitals to survive in the increasingly fierce healthcare market competition. However, it may be difficult for university hospitals to recruit and retain technically efficient surgeons because their missions include not only clinical services but also teaching and research. The authors hypothesized that technically efficient surgeons do not continue to work in a university hospital.

Methods: The authors collected data from all the surgical procedures performed at Teikyo University Hospital from April 1 through September 30 in 2013-2017. The dependent variable was defined as a length of employment of each surgeon. It was calculated as the number of years when a surgeon performed surgery as a senior surgeon at Teikyo University Hospital.

Output-oriented Charnes-Cooper-Rhodes model of data envelopment analysis was employed to calculate each surgeon's technical efficiency score. Inputs were defined as (1) the number of medical doctors who assisted surgery, and (2) the time of surgical operation from skin incision to skin closure (surgical time). The output was defined as the surgical fee for each surgery.

Six control variables that are available to us and may influence surgeons' length of employment were selected; experience, medical school, surgical volume, gender, and academic rank (professor, associate professor). Multiple regression analysis using ordinary least squares and ordered logit models was performed. A p-value $<0.05$ was considered statistically significant.
Results: The authors analyzed total 13,911 surgical cases in 30month study period from 2013 through 2017. Efficiency scores were calculated for all of them in each year, and the mean of the efficiency scores for each surgeon was calculated. Two hundred and eleven surgeons were analyzed. The results were shown in TABLE. Efficiency scores had significantly negative association with length of employment ( $p=0.011$ and 0.020). Experience and surgical volume had significantly positive association with length of employment $(p=0.000$ and $p=0.000$, respectively in both models). The other coefficients of control variables were insignificant.

Discussion: From our ordinary least squares and ordered logit models multiple regression analysis, we demonstrated that technically efficient surgeons have shorter length of employment at a university hospital. The longer their experiences were and the larger their surgical volumes were, the longer they work in a university hospital. Their medical school, gender, or academic ranks did not have any significant predictive values for their length of employment at a university hospital. Both statistical models reached the same results. This finding demonstrated that a university hospital cannot retain technically efficient surgeons after controlling their experience, surgical volumes, academic ranks, medical schools and gender. This is the first study that evaluated the relationship between surgeons' technical efficiency and their length of employment at a university hospital.

Conclusion: A university hospital cannot retain technically efficient surgeons.

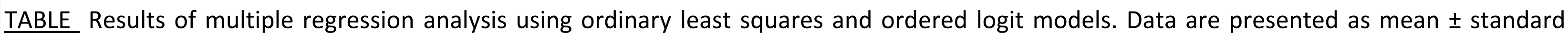
error. ${ }^{*}$ indicates that the coefficient is significantly different from zero $(p<0.05)$.

\begin{tabular}{|c|c|c|c|c|}
\hline & \multicolumn{4}{|c|}{ Dependent variable: Length of employment (years) } \\
\hline & \multicolumn{2}{|c|}{ Ordinary least squares } & \multicolumn{2}{|c|}{ Ordered logit } \\
\hline & Coefficients & $p$-value & Coefficients & p-value \\
\hline Efficiency Score * & $-1.418 \pm 0.549$ & 0.011 & $-1.974 \pm 0.847$ & 0.020 \\
\hline Experience $*$ & $0.059 \pm 0.014$ & 0.000 & $0.086 \pm 0.023$ & 0.000 \\
\hline Medical School & $-0.181 \pm 0.238$ & 0.448 & $-0.201 \pm 0.367$ & 0.584 \\
\hline Surgical Volume * & $0.022 \pm 0.006$ & 0.000 & $0.036 \pm 0.009$ & 0.000 \\
\hline Gender & $-0.027 \pm 0.300$ & 0.928 & $0.047 \pm 0.433$ & 0.913 \\
\hline Rank (Professor) & $-0.345 \pm 0.355$ & 0.333 & $-0.462 \pm 0.530$ & 0.384 \\
\hline Rank (Associate Professor) & $0.310 \pm 0.313$ & 0.323 & $0.414 \pm 0.472$ & 0.380 \\
\hline
\end{tabular}

